Probleme insbesondere daraus, daß verschiedene Diskriminierungsverbote der SRK (vgl. Art. 24 Abs. 1 lit.b, 26 Abs. 2, 42 Abs. 1, 2, 227 SRK) in Verbindung mit der Vorrangklausel zugunsten der SRK (Art. 4 Abs. 6 Annex IX SRK) die EWG-Staaten zwingen könnten, den Grundsatz der Inländergleichbehandlung im Zuständigkeitsbereich der Gemeinschaft auch auf Staatsangehörige von Nicht-EG-Staaten auszudehnen. Ederer versucht hier, eine Anerkennung der Gemeinschaftsrechtsordnung als Sonderrégime aus der - insoweit lapidaren - Vorschrift des Art. 311 Abs. 5 SRK abzuleiten. Jedoch gilt hier wie im Gesamtverhältnis zwischen EWG und SRK, daß erst das Inkrafttreten der Konvention aufzeigen wird, inwieweit materielle Inkompatibilitäten zur Gemeinschaft bestehen und lösbar sind.

Insgesamt besticht Ederers Arbeit durch die Komplexität ihrer Analyse und den Reichtum des ausgewerteten Materials.

Eine bessere drucktechnische. Ausgestaltung wäre ihr daher zu wünschen gewesen.

Marco Núñez-Müller

\title{
Arnd Bernaerts
}

\section{Bernaerts' Guide to the Law of the Sea}

The 1982 United Nations Convention (with full text of the Convention, Final Act and Index)

Coulsdon, Surrey: Fairplay Publications Ltd., 1988, 361 S., kartoniert, \$ 48,-

Wohl kaum ein anderes Gebiet des Völkerrechtes könnte in naher Zukunft solche Bedeutung erlangen wie das des Seerechtes.

Themen wie Tiefseebergbau und Meeresverschmutzung sind gerade in jüngster Zeit in den Vordergrund des öffentlichen Interesses gestoßen. Die Genfer UN-Seerechtskonvention von 1982 könnte auf diese Fragen Antworten geben und bei der Verständigung einen großen Beitrag leisten.

Dort hat man sich u.a. über Themen wie ausschließliche Wirtschaftszone und insbesondere über die Ausbeutung des Meeresbodens verständigt.

Zwar ist diese Konvention noch nicht in Kraft, doch gilt sie bereits heute als ein großes politisches Dokument, welches eine signifikante und beeinflußende Rolle auf die Entwicklung der Beziehungen zwischen Staaten hat.

Daher ist es auch nicht verwunderlich, daß insbesondere im europäischen Raum zahlreiche Neuerscheinungen auf dem Gebiet des Seerechtes bzw. der III. Seerechtskonvention veröffentlicht werden. Aus dieser Vielzahl fällt jedoch ein Buch aufgrund seiner neuartigen Konzeption ganz besonders ins Auge.

Es handelt sich um Bernaerts' Guide to the Law of the Sea, welches im Herbst 1988 in englischer Sprache erschienen ist. 
Der Rechtsanwalt und Diplom-Nautiker Bernaerts versteht es, seine Erfahrungen als Praktiker mit der nüchternen Analyse des Juristen zu verbinden. Gerade das kommt dem Leser bei der Behandlung eines solche komplexen Themas sehr zugute.

Bernaerts scheint der erste zu sein, der versucht hat, die Konvention in ihre Einzelstücke zu zerteilen und dann die jeweiligen Kapitel in Text und Graphiken zusammenzuf assen.

Es ist genau jene denkbar einfache Konzeption, welches dieses Buch sowohl für Neueinsteiger als auch für diejenigen, die sich intensiv mit dieser Konvention beschäftigen so nützlich macht.

Dieses wird auch insbesondere in dem Vorwort von dem Under-Secretary-General und Special Representative of the Secretary General of the United Nations for the Law of the Sea, Satya N. Nandan, hervorgehoben. So qualifiziert er das Buch - aufgrund seiner Zugänglichkeit - als unschätzbaren Führer für das Verständnis und wertvolle Ergänzung der III. Seerechtskonvention der Vereinten Nationen. Dem mag man nur zustimmen.

Das Buch besteht aus drei Teilen: Einer zwanzigseitigen Einführung zum historischen Hintergrund und der Entwicklung etc., einem "Guide" (ca. 80 Seiten) und einer Kommentierung der wichtigsten Überbegriffe und Regelungsbereiche der Konvention (ca. 30 Seiten). Daran schließt sich der Konventionstext, der Final Act und ein sehr übersichtlicher Index mit über 2000 Stichworten.

Das Kemstück ist jedoch der "Guide", auf den nun näher eingegangen werden soll.

Der "Guide" ist quasi ein Führer, der den Leser durch das Strickwerk der 320 Artikel, die neun Anhänge und den Final Act der Konvention führen soll.

Auf der linken Seite wird der Konventionstext durch eine Zusammenfassung aller einschlägigen Artikel zu dem jeweiligen Stichwort erklärt. Die Erklärung der Begriffe erfolgt somit immer anhand der Konvention und durch Verweise auf die Artikel.

Auf der rechten Seite schließt sich nun die graphische Darstellung an. In ihr werden die Beziehungen untereinander verdeutlicht und die wichtigsten Ergebnisse zusammengefaßt.

Durch diese Entzerrung erhält der "Guide" seine hervorragende Übersichtlichkeit, wodurch das Verständnis und die Arbeit erheblich erleichtert werden.

Besonders lobenswert ist die graphische Zusammenfassung der behandelten Abschnitte. Obwohl es sich - wohl eher aus drucktechnischen Gründen - nur um reine Strichgraphiken handelt, büßen sie kaum an ihrer Plastizität ein.

Ein möglicher Kritikpunkt wären vielleicht die fehlenden Literatur- oder Vertiefungshinweise. Jedoch würde man wohl das Konzept nicht mehr durchhalten und das Anliegen sprengen, wenn man - hinsichtlich der über 20 Regelungsbereiche - die Flut der Literatur zu diesem Gebiet berücksichtigen müßte. Eine mögliche 2. Auflage sollte aber dem interessierten Leser diesbezüglich etwas mehr entgegenkommen.

Bernaerts bemüht sich immer um eine deutliche Sprache und vermeidet so Verwirrungen, die bei der Behandlung eines solch komplexen Themenkreises unweigerlich auftreten. Er weicht jedoch nicht von der korrekten juristischen Wortwahl ab.

So prägt er daher notwendigerweise bei der Behandlung des Kontinentalsockels ("The Continental Shelf") einen neuen Begriff: 
Bei der Unterscheidung zwischen dem Meeresboden der ausschließlichen Wirtschaftszone ("sea-bed of the exclusive economic zone") und dem Meeresboden jenseits der Grenzen der ausschließlichen Wirtschaftszone ("sea-bed beyond the limits of the exclusive economic zone") bezeichnet er ersteren als "primary sea-bed", den zweiten als "outer shelf".

Die Notwendigkeit dieser Begriffe besteht darin, daß für das "primary sea-bed" die Art. $56 \mathrm{ff}$. und $76 \mathrm{ff}$., für den "outer shelf" jedoch nur die Art. 76 ff. der Konvention einschlägig sind.

Bernaerts hat durch diese Begriffe daher eine saubere und verständliche Abgrenzung und Unterscheidung geschaffen.

Durch die Konzeption wird somit das Buch ein geeignetes Vademecum für jeden, der sich mit dem Seerecht oder der III. UN-Seerechtskonvention beschäftigt oder beschäftigen will.

Die Veröffentlichung einer deutschen Fassung des "Guide" wäre sehr zu begrüßen. Aber darauf sollte man nicht warten, denn sechs Jahre nach dem Ende der III. UN-Seerechtskonferenz liegt leider immer noch keine offizielle deutsche Fassung der Seerechtskonvention für eine interessierte Öffentlichkeit vor.

Michael Bonefeld

Guy Kirsch, Klaus Mackscheidt

China. Ordnungspolitik in einem konfuzianischen Land

Nomos Verlagsgesellschaft, Baden-Baden 1988, 128 S., DM 37,-

The Chinese People's Republic, comprising almost all of Chinese ethnicity in Asia, has also remained the principal theatre of perennial attempts at large-scale adaptation of China's vast, still largely traditional society to the pressures of "modernity" as chiefly represented by industrial capitalism. Recognition that old homegrown ways would no longer serve has often provoked a marked assiduity to absorb foreign ways and means for the betterment of a new China. Thus numerous academic luminaries from the West travelled to early Republican China in the beginning of the 1920s to disseminate their teachings, not unlike their spiritual brethren who had previously tried to spred the Word among the "heathen Chinese".

Deng Xiaoping whose reform policies have now superseded the doctrinal rampages of the late Mao Zedong has again paved the way for a considerable flow into China of foreign ideas and their propounders, and once more the attempt is made to import foreign elements that may help to accelarate the modernisation of Chinese society.

In view of China's marked distinctness as compared with differences, say, between nations of Europe and North America, it may be suspected that many of those who have displayed their intellectual wares in China after 1979 were not in a position to prepare themselves 